\title{
BMJ Open The Celiac Disease and Diabetes- Dietary Intervention and Evaluation Trial (CD-DIET) protocol: a randomised controlled study to evaluate treatment of asymptomatic coeliac disease in type 1 diabetes
}

\begin{abstract}
Farid H Mahmud, ${ }^{1}$ Emilia N De Melo, ${ }^{1}$ Karima Noordin, ${ }^{1}$ Esther Assor, ${ }^{1}$ Kamaljeet Sahota, ${ }^{1}$ Jolie Davies-Shaw, ${ }^{1}$ Ernest Cutz, ${ }^{2}$ Gino Somers, ${ }^{2}$ Margaret Lawson, ${ }^{3}$ David R Mack, ${ }^{4}$ Patricia Gallego, ${ }^{5}$ Charlotte McDonald, ${ }^{6}$ Melanie D Beaton, ${ }^{7}$ Kevin Bax, ${ }^{8}$ Fred Saibil, ${ }^{9}$ Jeremy Gilbert, ${ }^{10}$ Susan Kirsch, ${ }^{11}$ Bruce A Perkins, ${ }^{12}$ Maria Cino, ${ }^{13}$ Eva Szentgyorgyi, ${ }^{14}$ Dror Koltin, ${ }^{15}$ Amish Parikh, ${ }^{15}$ Geetha Mukerji, ${ }^{16}$ Andrew Advani, ${ }^{17}$ Olivia Lou, ${ }^{18}$ Margaret A Marcon ${ }^{19}$
\end{abstract}

To cite: Mahmud FH, De Melo EN, Noordin K, et al. The Celiac Disease and Diabetes-Dietary Intervention and Evaluation Trial (CDDIET) protocol: a randomised controlled study to evaluate treatment of asymptomatic coeliac disease in type 1 diabetes. BMJ Open 2015;5: e008097. doi:10.1136/ bmjopen-2015-008097

- Prepublication history for this paper is available online. To view these files please visit the journal online (http://dx.doi.org/10.1136/ bmjopen-2015-008097).

Received 3 March 2015 Revised 9 April 2015 Accepted 12 April 2015

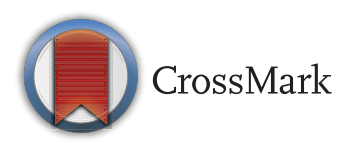

For numbered affiliations see end of article.

Correspondence to Dr Farid H Mahmud; Farid.mahmud@sickkids.ca

\section{ABSTRACT}

Introduction: Coeliac disease (CD) is an autoimmune condition characterised by gluten-induced intestinal inflammation, and observed at a 5-10 fold greater prevalence in type 1 diabetes. While universal screening for $C D$ in patients with diabetes is frequently advocated, objective data is limited as to benefits on diabetes control, bone health or quality of life related to the adoption of a gluten-free diet (GFD) in the large proportion of patients with diabetes with asymptomatic CD. The Celiac Disease and Diabetes-Dietary Intervention and Evaluation Trial (CD-DIET) study is a multicenter, randomised controlled trial to evaluate the efficacy and safety of a GFD in patients with type 1 diabetes with asymptomatic CD.

Methods and analysis: Children and adults (8-45 years) with type 1 diabetes will be screened for asymptomatic CD. Eligible patients with biopsy-proven CD will be randomly assigned in a $1: 1$ ratio to treatment with a GFD for 1 year, or continue with a gluten-containing diet. The primary outcome will evaluate the impact of the GFD on change in glycated haemoglobin. Secondary outcomes will evaluate changes in bone mineral density, blood glucose variability and health-related quality of life between GFD-treated and the regular diet group over a 1-year period. The study was initiated in 2012 and has subsequently expanded to multiple paediatric and adult centres in Ontario, Canada.

Ethics and dissemination: The findings from this study will provide high-quality evidence as to the impact of GFD treatment on glycaemic control and complications in asymptomatic children and adults with CD and type 1 diabetes.

Trial registration number: NCT01566110.

\section{Strengths and limitations of this study}

- The Celiac Disease and Diabetes-Dietary Intervention and Evaluation Trial (CD-DIET) study will provide high-quality data to clarify the impact of a gluten-free diet on clinically relevant outcomes including metabolic control (glycated haemoglobin, $\mathrm{HbA1C}$ ), bone health, glycaemic variability and quality of life in children and adults with type 1 diabetes and asymptomatic coeliac disease (CD).

- This is a randomised, multicentered, dietary intervention study in paediatric and adult diabetes centres across Ontario, Canada, with subjective and objective dietary assessment.

- Despite higher CD prevalence in patients with type 1 diabetes $(5-7 \%)$, a challenge of this study relates to the large number of individuals who will need to be screened to identify a cohort without symptoms to enter the randomised control dietary study.

\section{INTRODUCTION}

Coeliac disease (CD) is an autoimmune disease characterised by gastrointestinal inflammation caused by ingestion of gluten. ${ }^{1}$ While CD prevalence is near $1 \%$ in the general population, ${ }^{2}$ in type 1 diabetes, CD prevalence ranges from $3 \%$ to $16 \%^{3}$ in children, and from $1.4 \%$ to $6.8 \%$ in adults. ${ }^{4-6}$ As comorbid autoimmune diseases, type 1 diabetes and CD share a common genetic basis, ${ }^{7}$ and affected individuals may require lifelong lifestyle and dietary modification 
with adoption of a gluten-free diet (GFD). Additionally, recent epidemiological studies describe increased mortality and higher microvascular complication (nephropathy and retinopathy) rates in adult patients diagnosed with both $\mathrm{CD}$ and type 1 diabetes, suggestive that these patients represent a distinct risk group. ${ }^{8}{ }^{9}$ Despite the known association and potential therapeutic implications, there is a paucity of high-quality evidence, and few clinical trials to inform clinicians as to optimal strategies for screening and treatment for CD in the context of type 1 diabetes, especially in the high proportion (60$70 \%$ ) of asymptomatic patients. ${ }^{35}$

With regard to metabolic control, research in patients with type 1 diabetes with CD is variable, largely paediatric centred, and lacking objective assessment of glycaemic variability. In the largest group of children evaluated prospectively, with mostly asymptomatic CD, an increase in glycated haemoglobin (HbAlc) of $0.6 \%$ was observed after 1 year of GFD treatment, ${ }^{10}$ while other reports have described decreases and no change in HbAlc after GFD adoption. ${ }^{11-15}$ In a report of type 1 diabetes adults at CD diagnosis and after GFD treatment, no significant difference in $\mathrm{HbAlc}^{16}$ was seen, although there was a trend towards improved metabolic control in GFD-adherent patients. ${ }^{17}$ In asymptomatic patients with both $\mathrm{CD}$ and type 1 diabetes who selfselected a GFD or regular diet, no differences in HbAlc or frequency of hypoglycaemic events were observed after 2 years of follow-up. However, diet adherence was inconsistent in both groups. ${ }^{18}$ These reports highlight the importance of dietary rigour and quality in the evaluation of the impact of the GFD in metabolic control and hypoglycaemic events in patients with type 1 diabetes and CD.

While metabolic control remains a key outcome in diabetes care, impaired bone health represents a silent and insidious complication of $\mathrm{CD}$ in diabetes. $\mathrm{CD}$ alone is associated with increased risk of osteoporosis ${ }^{19}$ and fractures in adults, and impaired bone mineralisation in paediatrics. $^{20}$ In children with type 1 diabetes and untreated CD, $62.5 \%$ were shown to have had lumbar spine bone mineral density (BMD) Z-score lower than 1 SD compared with adequate lumbar spine BMD Z-scores reported in type 1 diabetes controls. ${ }^{21}$ Additionally, in children with type 1 diabetes and CD, lumbar spine BMD Z-scores were similar to matched type 1 diabetes controls, although dual diagnosis patients had higher parathyroid hormone (PTH) levels and urine bone remodelling markers. ${ }^{22}$ A single adult study evaluated women with type 1 diabetes for BMD, and reduced mean lumbar spine BMD Z-score was seen in women with type 1 diabetes and $\mathrm{CD}$ in comparison with patients with type 1 diabetes alone, ${ }^{23}$ underscoring the significance of bone health in type 1 diabetes and asymptomatic CD.

In studies of chronic conditions such as diabetes, which require daily monitoring and insulin administration, it is also essential to evaluate the additional impact of the diagnosis of $\mathrm{CD}$ on lifestyle and quality of life (QOL) in children and adults with type 1 diabetes. Type 1 diabetes and its management alone impact QOL throughout the patient's lifespan. ${ }^{24}$ Moreover, adoption of a GFD is associated with difficulties in social activities, eating out and travelling ${ }^{25}{ }^{26}$ in children and adults with CD. Children with type 1 diabetes and CD report limited availability of gluten-free products at school and restaurants, with dietary arrangements outside of the home reported as the most common issues related to GFD adoption. ${ }^{27}$ Moreover, $68 \%$ of the adults treated with GFD describe reduced enjoyment of food, and $27 \%$ who were asymptomatic regretted being diagnosed. ${ }^{28}$ Studies largely show QOL improvements after dietary treatment in symptomatic patients. ${ }^{29}$ In children, QOL score improvements after treatment are higher in patients who presented with symptoms at diagnosis than in asymptomatic patients. ${ }^{30}$ Parents, overall, report lower scores than their children, particularly parents of patients with type 1 diabetes and $\mathrm{CD}$ reporting lower social functioning scores for their children than parents of children with type 1 diabetes alone. ${ }^{31}{ }^{32}$ Similarly, adults with type 1 diabetes and CD report lower QOL scores compared with patients with type 1 diabetes alone, ${ }^{33}$ and adult patients with $\mathrm{CD}$ who present with symptoms adherent with the GFD show significant improvements in health-related QOL, whereas asymptomatic screen-detected cases describe a decline in selfperceived health. ${ }^{29}$ A longer term duration of treatment likely moderates the impact on patient QOL, as adaptation to the GFD for more than 5 years was associated with better QOL scores in adult patients with $\mathrm{CD}{ }^{34}$

Given the limitations in existing evidence for screening and initiating treatment in asymptomatic $\mathrm{CD}$ in type 1 diabetes, the CD-DIET study was designed as a prospective controlled trial to inform if the benefits of adopting a GFD outweigh the challenges of managing a significant change in diet and lifestyle. The hypotheses to be tested are that GFD-treated, asymptomatic patients with $\mathrm{CD}$ as well as type 1 diabetes will differ with respect to changes in HbAlc, BMD, frequency of hypoglycaemic events, and quality of life over a 1-year period from the regular, gluten-containing diet (GCD) group.

\section{METHODS AND ANALYSIS}

The proposal to design a large-scale clinical trial to evaluate the impact of asymptomatic CD and type 1 diabetes was submitted as a potential study for review in 2010 as part of the JDRF-Canadian Clinical Trial Network (JDRF-CGTN) call for proposals. Created in 2009, JDRF-CCTN is a shared investment by the Government of Canada (FedDev Ontario) and the JDRF. The CCTN was established to create a strong clinical research network to develop and conduct leading-edge clinical trials in type 1 diabetes and its complications. After extensive review, the study was initiated at a single site in 2012 with additional expansion taking place at 
over 18 different academic and community-based diabetes clinics throughout Ontario, Canada. Research Ethics Board approval was obtained from all participating sites, and regular protocol modifications and amendments will be reported to them. All data collected will be kept confidential and assured by the monitoring institution.

\section{Objectives}

The primary outcome is to evaluate the safety and efficacy of 1-year GFD treatment on HbAlc levels in patients with type 1 diabetes with asymptomatic biopsyproven CD. Secondary outcomes will assess the effect of a 1-year GFD treatment on the lumbar spine BMD, frequency of hypoglycaemic events, blood glucose (BG) variability, QOL and health perception.

\section{Study design}

The study has two distinct phases, screening and intervention. Patients aged 8-45 years with an established diagnosis of type 1 diabetes equal to or greater than 1 year will be screened for asymptomatic CD. Asymptomatic persons, who screen positive for CD with positive serology results and confirmed with duodenal biopsy (Modified Marsh classification score 2 or greater) will be randomly assigned to treatment with GFD for 1 year, or continue with their usual GCD, with 1:1 ratio. Randomisation will be performed centrally, using a computer-generated randomisation schedule, stratified by age.

\section{Study population and screening phase}

Eligibility criteria for the intervention trial are described in box 1. Individuals will be contacted through direct encounters at participating diabetes centres, or contact study staff through study advertisements or recruitment events. Potential participants will be contacted, and informed consent/assent will be obtained. Anthropometric measurements (eg, height, weight), recent HbAlc, age and date of type 1 diabetes diagnosis, history of previous CD screening, insulin regime and diabetes complications will be collected. Changes in weight or BMI below 3rd centile, or crossing of 2 growth percentiles in persons younger than 18 years, or an unintentional weight loss of greater than $10 \%$ of the weight of the past 3 months in adults ( $>18$ years) will be considered symptomatic and result in a patient exclusion.

Symptoms will be assessed through completion of the Gastrointestinal Symptom Scale (GISS) questionnaire, ${ }^{35}$ a self-reported 9-item symptom questionnaire, which includes the following elements: absence of symptoms, presence of upper and lower abdominal pain, loss of appetite, nausea, loose or hard stool, vomiting, pain or cramps related to menses or recent viral illness and a visual analogue scale (VAS) from 0 to $100 \mathrm{~mm}$ for severity evaluation. A patient who reports less than three symptoms during the past week (pain or cramps related to menses or recent viral illness will not be counted as a symptom) and a VAS less than $30 \mathrm{~mm}$ in severity will be considered asymptomatic.

If asymptomatic, blood samples will be obtained for haemoglobin $(\mathrm{Hb})$ levels and $\mathrm{CD}$ screening with tissue transglutaminase immunoglobulin A (TTG-IgA). Patients with $\mathrm{CD}$ positive serology will have the $\mathrm{Hb}$ level confirmed above the threshold for anaemia specific for age and gender criteria: females-under 10 years $\mathrm{Hb}<110 \mathrm{~g} / \mathrm{L}$ or haematocrit $<0.33 ; 10-18$ years $\mathrm{Hb}<115 \mathrm{~g} / \mathrm{L}$ or haematocrit $<0.34$; above 18 years $\mathrm{Hb}<120 \mathrm{~g} / \mathrm{L}$ or haematocrit $<0.36$; males-under 10 years $\mathrm{Hb}<110 \mathrm{~g} / \mathrm{L}$ or haematocrit $<0.33$; $10-14$ years $\mathrm{Hb}<115 \mathrm{~g} / \mathrm{L}$ or haematocrit $<0.34$; $15-18$ years $\mathrm{Hb}<120 \mathrm{~g} / \mathrm{L}$ or haematocrit $<0.36$; above 18 years $\mathrm{Hb}<130 \mathrm{~g} / \mathrm{L}$ or haematocrit $<0.40{ }^{36}$

Regional gastroenterologists engaged with the study will perform a consultation to review symptoms and perform endoscopy with duodenal biopsy. Symptomatic patients will be excluded from the study and will be managed clinically. Asymptomatic patients with a positive biopsy as per Modified Marsh Score of 2 or greater with central pathology confirmation will be considered positive for $\mathrm{CD}$, participants will be eligible for randomisation and participation in the intervention phase (figure 1 and box 1).

\section{Intervention}

Baseline visit

Participants randomised to GFD or to GCD will be followed for 1 year and seen every 3 months by a dietitian for the duration of the study for a total of five visits. All study dietitians will receive training by the lead site dietitian with standardised educational material and detailed steps for the diet interview at each visit to ensure quality

Box 1 Summary of eligibility criteria for intervention phase

Inclusion criteria

- Age 8-45 years

- Type 1 diabetes duration $\geq 1$ year

- Positive coeliac disease (CD) screen serology-tissue transglutaminase immunoglobulin A (TTG-IgA)

- Positive duodenal biopsy with a Modified Marsh score $\geq 2$

- Ability of the subject or a legal representative to speak and read English or French

Exclusion criteria

- Previous CD diagnosis

- Following gluten-free diet (GFD)

- CD-related gastrointestinal symptoms

- Abnormal growth or weight loss

- Anaemia

- History of osteoporosis

- Recurrent apthous ulcers or dermatitis herpetiformis

- Pregnancy or breastfeeding

- Participating in another intervention study

- Participant has any conditions which the investigator considers will interfere with the individual's ability to participate in the study 


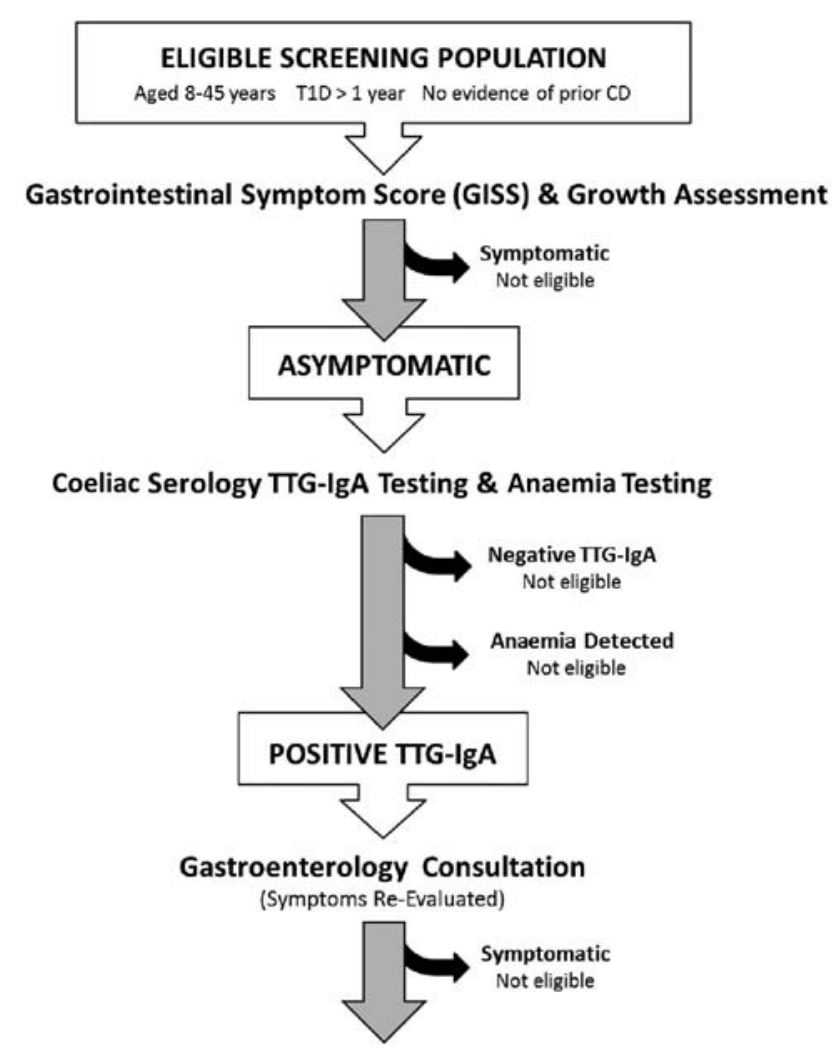

Biopsy Procedure \& Pathological Review

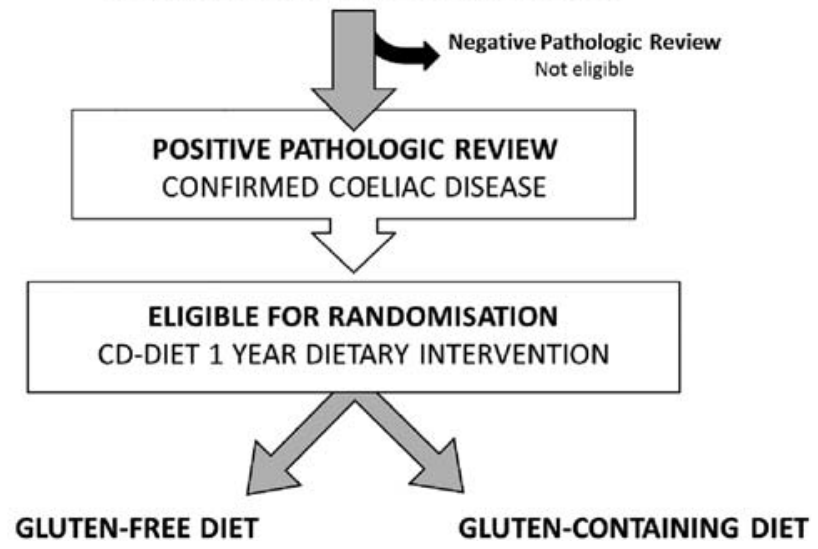

Figure 1 Flow diagram illustrating screening phase and eligibility for intervention phase for Celiac Disease and Diabetes-Dietary Intervention and Evaluation Trial (CD-DIET) study (T1D, type 1 diabetes; TTG-IgA, tissue transglutaminase immunoglobulin $\mathrm{A}$ ).

and consistency of dietary education. For both groups, the study dietitian will address follow-up questions, barriers to dietary adherence, and document adverse events.

Patients assigned to the GFD group will meet with a dietitian for a nutritional assessment and completion of GFD education. For the education component, the manual 'Your Guide to Well-being: Managing Celiac Disease and Diabetes' specifically designed for this study, the 'Pocket Dictionary of Ingredients' and the 'Checking Ingredient List' by the Canadian Celiac Association will be used.
Individuals assigned to the GCD will undergo a comprehensive nutritional assessment of their current usual gluten intake in the context of managing their diabetes.

At the initial visit, previous medical history including bone health, autoimmune diseases and family history of $\mathrm{CD}$ and type 1 diabetes will be documented. Individual diabetes history and treatment details, vitamins and supplements will also be assessed.

Participants in both groups will complete food records for 3 days (two weekdays and one weekend day) which will be analysed to assess for dietary deficiencies or imbalances, using a nutritional database software program (ESHA, http:/ /www.esha.com), as well as gluten content.

A follow-up phone call at 4 weeks will be placed for each group to provide a nutritional and psychosocial support, clarify concepts of the GFD in this group, and assess for adherence and barriers to following GFD or GCD.

\section{Follow-up visits}

For the GFD group, the interview in the follow-up visits will address any gaps in education; provide nutritional support and reinforcement of GFD topics as required. For the GCD group, the interview will aim to support current dietary management.

Participants in both groups will complete food records for 3 days for 6-month and 12-month visits, which will be analysed to assess for dietary deficiencies or imbalances using ESHA as well as gluten content. Results will be reviewed with the participants at their following visits (3 and 9 months, respectively) for imbalances and deficiencies, and a plan for issues or concerns will be implemented. A 'Typical Day Intake' will be completed to assess for gluten exclusion or inclusion at 3-month and 9-month visits.

At all follow-up visits, participants in both study groups will be interviewed to assess adverse events, changes in insulin regimen or in non-insulin medications, including supplements and vitamins, and diabetes complications. All adverse events will be reported, and if it is determined that the participants should be withdrawn from the study, they will be required to complete a final study visit (figure 2).

\section{Adherence}

Dietary adherence will be assessed at each visit following the baseline visit. Adherence assessment will be classified as subjective and objective. Subjective evaluation will include a designed dietary interview which, for the GFD group, will include specifically the participant's ability to distinguish gluten-free food from gluten-containing food, cross-contamination and maintenance of GFD outside of the home and in social situations. The dietary interview for the GCD will assess for inclusion or exclusion of gluten in the diet. Participants will complete a 'self-reported' questionnaire designed to evaluate their comfort (both groups) and understanding (GFD group) with their assigned diet. At the end of the dietary interview, the dietitian will rank patients' adherence to their 
Figure 2 Intervention phaseoutcomes and safety (BMD, bone mineral density; $\mathrm{HbA1c}$, glycated haemoglobin; TTG-IgA, tissue transglutaminase immunoglobulin A).

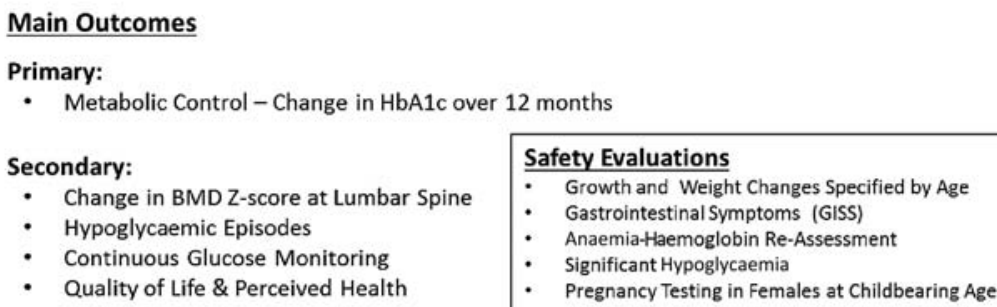

Dietary Intervention assigned diet. Objective assessment of adherence will include food records collected and a typical day's intake for both groups in addition to gluten quantification. TTG-IgA titres will be measured at 6 -month and 12-month visits to assess GFD/GCD compliance. Additionally, the GFD group will complete the Celiac Dietary Adherence Test questionnaire, to evaluate symptom and gluten intake at every visit after randomisation. ${ }^{37}$

An optional telephone contact at 7 months will be conducted only for participants who are found to be non-adherent to the assigned diet.

\section{Outcomes and Safety}

The primary outcome in CD-DIET study is change in metabolic control, which will be assessed at every visit by HbA1c. HbA1c will be measured by standardised highperformance liquid chromatography assays using International Federation of Clinical Chemistry and Laboratory Medicine reference guidelines.

As a secondary outcome, bone health will be evaluated at baseline and 12-month visits. Data collection on the first visit will evaluate family history of osteoporosis, individual history of fracture, smoking habits and ethnicity. Self-Rated Pubertal Staging questionnaires will also be collected for participants younger than 16 years of age. Laboratory measurements will be performed at the same visits for serum calcium, phosphate, magnesium, PTH and vitamin $\mathrm{D}$, as well as BMD measurement. BMD will be measured at lumbar spine (L1-L4) in the anteroposterior direction using Dual-Energy X-ray Absorptiometry
(DXA) scan. All measurements will be classified as Z-scores and compared with established population reference data for age, sex and ethnicity in accordance with recommendations by International Society for Clinical Densitometry 2007. DXA will be conducted in qualified centres, and all densitometers will be calibrated at the start and yearly for the duration of the study using a spine phantom (Bio-Imaging Bona Fide Spine Phantom). Equivalent DXA manufacturer models (Hologic or Lunar Prodigy) values will be assured by the Hologic 12.4 normative database, and values will be presented as age-specific and sex-specific Z-scores. Bone age will also be assessed using the left hand and wrist radiograph in individuals younger than 16 years of age, and it will be evaluated centrally.

QOL will be assessed as secondary outcome at baseline, 6-month and 12-month visits using the paediatric Quality of Life (PedsQL) Inventory Generic Core Scale (V.4.0) and the Diabetes Module (V.3.0). Both validated questionnaires for children, adolescents and adults with type 1 diabetes. ${ }^{38}$

Hypoglycaemic episodes frequency and severity will be assessed at each visit. Additionally, continuous glucose monitoring (CGM) will be completed at baseline at 6-month and 12-month visits to evaluate asymptomatic hypoglycaemic events as well as patterns of BG excursion relating to postprandial peaks and timing of hypoglycaemic events. Individuals will use the Medtronic Guardian iPro2 CGM system for 7 days, with a minimum of 3 days, for blinded assessment of interstitial BG evaluations. 
At baseline and 12-month visits, for safety reasons, laboratory tests will be collected for ferritin, complete blood count, alanine transaminase, aspartate transaminase, low-density lipoprotein, high-density lipoprotein, total cholesterol, tryglicerides, urea, creatinine and urinary albumin. $\mathrm{Hb}$ level will be reassessed at 6 -month and 12-month visits.

Regular monitoring of height, weight and BMI, as well as gastrointestinal symptoms using the GISS questionnaire will be assessed at each follow-up visit, and participants will be considered symptomatic if criteria previously described in the screening phase are met for anthropometric measures. For the GISS, the individual will be considered symptomatic if three or more symptoms unrelated to menstruation or acute infectious illness are reported, and a VAS score greater than $30 \mathrm{~mm}$ is reported for the past week.

Pregnancy testing will be performed in all women of childbearing age at baseline, 6-month and 12-month visits with quantitative $\beta \mathrm{HCG}$, and at all visits in all female subjects who are attempting to get pregnant or suspect pregnancy. A positive pregnancy test will result in the individual's withdrawl.

Individuals from the GCD group who become symptomatic will be crossed over to the GFD group. Symptoms or other evidence of overt CD will be defined by at least one of the following: gastrointestinal symptoms using GISS, poor growth, significant symptomatic hypoglycaemic events or anaemia. The individuals who cross over to the GFD will complete an early withdrawal visit for outcome and safety measurements.

\section{Power calculation and statistics}

The outcome measure used to calculate sample size will be the change in HbA1c level from baseline to month 12. Based on previous work, in a non-randomised comparison of HbA1c levels over time in type 1 diabetes patients with asymptomatic CD who received a GFD versus patients with type 1 diabetes alone, an increase in HbAlc of $8.3 \pm 1.1 \%$ to $8.9 \pm 1.5 \%$ was observed. ${ }^{10}$ As such, 91 evaluable individuals per group are required to provide $80 \%$ power to detect a difference of at least $0.5 \%$ at the 0.05 level of significance. The enrolment of 200 participants will allow for a non-evaluable rate of about $10 \%$. To reach the recruitment target of 200 individuals, approximately 5000 patients with an established diagnosis of type 1 diabetes will be screened for asymptomatic CD. Efficacy analyses will be performed on the intent-to-treat population consisting of all randomised participants.

The primary efficacy outcome, the change in HbAlc at 12 months, will be analysed using an analysis of covariance (ANCOVA) model that includes adjustment for baseline HbAlc, age groups and investigative sites. Treatment effect will be quantified using the point estimate, the two-sided 95\% CI, and associated $\mathrm{p}$ value. Significant effect will be declared at the $5 \%$ significance level.
Severe hypoglycaemic episodes over time will be analysed as Poisson counts using the generalised estimating equations approach to account for correlation among repeated measures within an individual. The QOL data over time will be analysed by means of a linear mixed effects model including factors for treatment, investigative sites, age groups, and baseline QOL scores. BG variability and change in Z-score at the lumbar spine will be analysed using the ANCOVA approach. The Statistical Analysis System (SAS) procedures GENMOD, MIXED and GLM in SAS V.9.2, will be used to perform the respective analyses.

The CD-DIET study data safety and monitoring board will perform regular reviews of collected safety and efficacy data.

\section{ETHICS AND DISSEMINATION}

The CD-DIET study will contribute important information regarding treatment of asymptomatic patients with dual diagnosis and the effect of the GFD on diabetes control, bone health, QOL and CD complications. Furthermore, it is anticipated that the results will provide high-quality data to help clarify the question of whether universal screening for CD should be performed in children and adults with type 1 diabetes.

CD has a higher prevalence in patients with type 1 diabetes compared with the general population, but it is still a relatively uncommon condition diagnosed in a minority of patients with diabetes. As such, a challenge of this study relates to the large number of individuals who will need to be screened to identify a cohort without symptoms to enter the randomised control dietary study. The Canadian context to the study is important, as our national Canadian Diabetes Association 2013 guidelines do not recommend universal CD screening, rather suggests a targeted symptombased screening approach which recommends serological testing based solely on clinical symptoms including recurring gastrointestinal symptoms, poor weight gain, anaemia and unexplained frequent low blood sugars. ${ }^{39}$ This differs from other consensus-based guidelines for CD screening used in children, such as the International Society for Paediatric and Adolescent Diabetes $^{39-44}$ which recommends CD screening at type 1 diabetes diagnosis and annually for 5 years ${ }^{40}$; while in adults, recommendations are less specific. ${ }^{43}{ }^{44}$ From a regional perspective, the lack of $\mathrm{CD}$ screening in clinical practice in many diabetes clinics in Ontario is also related to the fact that costs of $\mathrm{CD}$ serologic screening tests are not covered by our provincial health plan in out-of-hospital laboratory facilities. ${ }^{45}$

An additional challenge relates to potential difficulties with individual adherence to their assigned dietary group. As such, significant effort was invested in a patent and family centred dietary experience supported by dietitians with extensive knowledge of diabetes, and a curriculum with subjective and objective assessment for 
dietary quality and gluten content. A de novo study dietary handbook was also created for the study, with sections focusing on adaption to the GFD highlighting situational approaches (eating out, travelling, sleepovers) and providing diabetes-specific nutritional tables for carbohydrates and glycaemic index. Additionally, a monthly subsidy $(\mathrm{CDN} \$ 100)$ is being provided to individuals who are randomised the GFD to recognise additional costs associated with the diet.

The CD-DIET study design is multicentre and will include both paediatric and adult patients. Engagement will take place with diabetes clinics with established clinical trial infrastructure and skilled and trained research staff. Furthermore, community-based resources will be used to search for eligible patients for screening such as educational seminars with healthcare professionals and advertisements to the target population using posters, brochures, websites, outreach community and educational events.

This article provides guidance towards the design of the CD-DIET study (http://www.celiacanddiabetes.com) with detailed descriptions of outcome measurements and instruments that will be used at each step of screening and dietary intervention phases. This study was initiated in 2012 with screening taking place at a single site. The study is now active at 18 paediatric and adult centres, and it is anticipated that results will be available by 2016 .

\section{Author affiliations}

${ }^{1}$ Department of Pediatrics, Division of Endocrinology, Hospital for Sick Children, University of Toronto, Toronto, Ontario, Canada

${ }^{2}$ Department of Pathology, Hospital for Sick Children, University of Toronto, Toronto, Ontario, Canada

${ }^{3}$ Division of Endocrinology and Metabolism, Children's Hospital of Eastern Ontario, Ottawa, Ontario, Canada

${ }^{4}$ Division of Gastroenterology, Hepatology and Nutrition, Children's Hospital of Eastern Ontario, Ottawa, Ontario, Canada

${ }^{5}$ Division of Endocrinology Paediatrics, London Health Sciences Centre, London, Ontario, Canada

${ }^{6}$ Division of Endocrinology and Metabolism, St. Joseph Health Care, London Health Sciences Centre, London, Ontario, Canada

${ }^{7}$ Division of Gastroenterology, London Health Sciences Centre, London, Ontario, Canada

${ }^{8}$ Pediatric Gastroenterology, Department of Pediatrics, Children's Hospital, London Health Sciences Centre, London, Ontario, Canada

${ }^{9}$ Division of Gastroenterology, Sunnybrook Health Sciences Centre, Toronto, Ontario, Canada

${ }^{10}$ Division of Endocrinology and Metabolism, Sunnybrook Health Sciences Centre, Toronto, Ontario, Canada

${ }^{11}$ Division of Endocrinology, Markham Stouffville Hospital, Toronto, Ontario, Canada

${ }^{12}$ Division of Endocrinology and Metabolism, Department of Medicine, University Health Network, University of Toronto, Toronto, Ontario, Canada

${ }^{13}$ Division of Gastroenterology, Toronto Western Hospital, University Health Network, University of Toronto, Toronto, Ontario, Canada

${ }^{14}$ Department of Pathology, University Health Network, University of Toronto,

Toronto, Ontario, Canada

${ }^{15}$ Division of Endocrinology, Trillium Health Partners, Mississauga, Ontario, Canada

${ }^{16}$ Division of Endocrinology, Women's College Hospital, Toronto, Ontario, Canada

${ }^{17}$ Division of Endocrinology, St. Michael's Hospital, Toronto, Ontario, Canada

${ }^{18}$ Juvenile Diabetes Research Foundation-Canadian Clinical Trials Network (JDRF-CCTN), Toronto, Ontario, Canada
${ }^{19}$ Division of Gastroenterology, Hepatology and Nutrition, Hospital for Sick Children, University of Toronto, Toronto, Ontario, Canada

Contributors FHM conceived and designed the research project, led its coordination, participated in data acquisition and drafted the initial manuscript. ENDM participated in the manuscript writing. KN participated in data acquisition and drafted the initial manuscript. EA reviewed the manuscript and contributed to the discussion. KS participated in data acquisition and reviewed the initial manuscript. JDS reviewed the manuscript. EC, GS, ML, DRM, PG, CM, MDB, KB, FS, JG, SK, BAP, MC, ES, DK, AP, GM and $A A$ participated in data acquisition and manuscript review. OL participated in the manuscript review. MAM participated in the design of the research project, its coordination, participated in data acquisition, and drafted the initial manuscript.

Funding This work is supported by Juvenile Diabetes Research FoundationCanadian Clinical Trials Network (JDRF-CCTN) grant number (CCTN 1103) and receives operating support from Robarts Clinical Trials Inc. for data monitoring, quality assurance and adverse events coordination.

Competing interests FHM has been a speaker without either honorarium or travel reimbursement at educational events sponsored by Animas Canada and the JDRF. The CD-DIET project was presented in abstract form at the ISPAD Meeting 2014.

\section{Patient consent Obtained.}

Ethics approval Research Ethics Board Hospital for Sick Children, University of Toronto, Toronto-ON, Canada.

Provenance and peer review Not commissioned; externally peer reviewed.

Data sharing statement All participating sites agreed in contract to data sharing with the primary investigating site and the sponsor. The access to the final trial dataset will be to the Primary Investigator and the Juvenile Diabetes Research Foundation-Canadian Clinical Trials Network (JDRF-CCTN).

Open Access This is an Open Access article distributed in accordance with the Creative Commons Attribution Non Commercial (CC BY-NC 4.0) license, which permits others to distribute, remix, adapt, build upon this work noncommercially, and license their derivative works on different terms, provided the original work is properly cited and the use is non-commercial. See: http:// creativecommons.org/licenses/by-nc/4.0/

\section{REFERENCES}

1. Farrell RJ, Kelly CP. Celiac sprue. N Engl J Med 2002;346:180-8.

2. Rubio-Tapia A, Ludvigsson JF, Brantner TL, et al. The prevalence of celiac disease in the United States. Am J Gastroenterol 2012;107:1538-44; quiz 1537, 1545.

3. Sud S, Marcon M, Assor E, et al. Celiac disease and pediatric type 1 diabetes: diagnostic and treatment dilemmas. Int $J$ Pediatr Endocrinol 2010;2010:161285.

4. Page SR, Lloyd CA, Hill PG, et al. The prevalence of coeliac disease in adult diabetes mellitus. QJM 1994;87:631-7.

5. Mahmud FH, Murray JA, Kudva YC, et al. Celiac disease in type 1 diabetes mellitus in a North American community: prevalence, serologic screening, and clinical features. Mayo Clin Proc 2005;80:1429-34.

6. Remes-Troche JM, Rios-Vaca A, Ramirez-Iglesias MT, et al. High prevalence of celiac disease in Mexican Mestizo adults with type 1 diabetes mellitus. J Clin Gastroenterol 2008;42:460-5.

7. Smyth DJ, Plagnol V, Walker NM, et al. Shared and distinct genetic variants in type 1 diabetes and celiac disease. $N$ Engl J Med 2008;359:2767-77.

8. Mollazadegan K, Fored M, Lundberg S, et al. Risk of renal disease in patients with both type 1 diabetes and coeliac disease. Diabetologia 2014;57:1339-45

9. Mollazadegan K, Kugelberg M, Montgomery SM, et al. A populationbased study of the risk of diabetic retinopathy in patients with type 1 diabetes and celiac disease. Diabetes Care 2013;36:316-21.

10. Sun S, Puttha R, Ghezaiel S, et al. The effect of biopsy-positive silent coeliac disease and treatment with a gluten-free diet on growth and glycaemic control in children with type 1 diabetes. Diabet Med 2009;26:1250-4.

11. Saadah OI, Zacharin M, O'Callaghan A, et al. Effect of gluten-free diet and adherence on growth and diabetic control in diabetics with coeliac disease. Arch Dis Child 2004;89:871-6. 
12. Abid N, McGlone O, Cardwell C, et al. Clinical and metabolic effects of gluten free diet in children with type 1 diabetes and coeliac disease. Pediatr Diabetes 2011;12:322-5.

13. Hansen D, Brock-Jacobsen B, Lund E, et al. Clinical benefit of a gluten-free diet in type 1 diabetic children with screening-detected celiac disease: a population-based screening study with 2 years' follow-up. Diabetes Care 2006;29:2452-6.

14. Sanchez-Albisua I, Wolf J, Neu A, et al. Coeliac disease in children with type 1 diabetes mellitus: the effect of the gluten-free diet. Diabet Med 2005;22:1079-82.

15. Taler I, Phillip M, Lebenthal Y, et al. Growth and metabolic control in patients with type 1 diabetes and celiac disease: a longitudinal observational case-control study. Pediatr Diabetes 2012;13:597-606.

16. Bakker SF, Tushuizen ME, von Blomberg ME, et al. Type 1 diabetes and celiac disease in adults: glycemic control and diabetic complications. Acta Diabetol 2013;50:319-24.

17. Leeds JS, Hopper AD, Hadjivassiliou M, et al. High prevalence of microvascular complications in adults with type 1 diabetes and newly diagnosed celiac disease. Diabetes Care 2011;34:2158-63.

18. Simmons $\mathrm{JH}$, Klingensmith GJ, McFann $\mathrm{K}$, et al. Celiac autoimmunity in children with type 1 diabetes: a two-year follow-up. $J$ Pediatr 2011;158:276-81.e1.

19. Viljamaa $\mathrm{M}$, Collin $\mathrm{P}$, Huhtala $\mathrm{H}$, et al. Is coeliac disease screening in risk groups justified? A fourteen-year follow-up with special focus on compliance and quality of life. Aliment Pharmacol Ther 2005;22:317-24.

20. Heikkila K, Pearce J, Maki M, et al. Celiac disease and bone fractures: a systematic review and meta-analysis. J Clin Endocrinol Metab 2015;100:25-34.

21. Diniz-Santos DR, Brandao F, Adan L, et al. Bone mineralization in young patients with type 1 diabetes mellitus and screening-identified evidence of celiac disease. Dig Dis Sci 2008;53:1240-5

22. Simmons $\mathrm{JH}$, Klingensmith GJ, McFann $\mathrm{K}$, et al. Impact of celiac autoimmunity on children with type 1 diabetes. $J$ Pediatr 2007:150:461-6.

23. Lunt $\mathrm{H}$, Florkowski $\mathrm{CM}$, Cook HB, et al. Bone mineral density, type 1 diabetes, and celiac disease. Diabetes Care 2001;24:791-2.

24. Jacobson AM, Braffett BH, Cleary PA, et al. The long-term effects of type 1 diabetes treatment and complications on health-related quality of life: a 23-year follow-up of the Diabetes Control and Complications/Epidemiology of Diabetes Interventions and Complications cohort. Diabetes Care 2013;36:3131-8.

25. Rashid M, Cranney A, Zarkadas M, et al. Celiac disease: evaluation of the diagnosis and dietary compliance in Canadian children. Pediatrics 2005;116:e754-9.

26. Zarkadas M, Dubois S, Maclsaac K, et al. Living with coeliac disease and a gluten-free diet: a Canadian perspective. J Hum Nutr Diet 2013;26:10-23.

27. Saukkonen T, Vaisanen S, Akerblom HK, et al. Coeliac disease in children and adolescents with type 1 diabetes: a study of growth, glycaemic control, and experiences of families. Acta Paediatr 2002;91:297-302.

28. Whitaker JK, West J, Holmes GK, et al. Patient perceptions of the burden of coeliac disease and its treatment in the UK. Aliment Pharmacol Ther 2009;29:1131-6.
29. Ukkola A, Maki M, Kurppa K, et al. Diet improves perception of health and well-being in symptomatic, but not asymptomatic, patients with celiac disease. Clin Gastroenterol Hepatol 2011:9:118-23.

30. Bystrom IM, Hollen E, Falth-Magnusson $\mathrm{K}$, et al. Health-related quality of life in children and adolescents with celiac disease: from the perspectives of children and parents. Gastroenterol Res Pract 2012;2012:986475

31. Sud S, Marcon M, Assor E, et al. Quality of life in children with diabetes and celiac disease: minimal impact of the 'double diagnosis'. Pediatr Diabetes 2012;13:163-9.

32. van Koppen EJ, Schweizer JJ, Csizmadia CG, et al. Long-term health and quality-of-life consequences of mass screening for childhood celiac disease: a 10-year follow-up study. Pediatrics 2009;123:e582-8.

33. Bakker SF, Pouwer F, Tushuizen ME, et al. Compromised quality of life in patients with both type 1 diabetes mellitus and coeliac disease. Diabet Med 2013;30:835-9.

34. Pulido O, Zarkadas M, Dubois S, et al. Clinical features and symptom recovery on a gluten-free diet in Canadian adults with celiac disease. Can J Gastroenterol 2013;27:449-53.

35. Wolfe F, Kong SX, Watson DJ. Gastrointestinal symptoms and health related quality of life in patients with arthritis. $J$ Rheumatol 2000;27:1373-8.

36. Mack DR, Langton C, Markowitz J, et al. Laboratory values for children with newly diagnosed inflammatory bowel disease. Pediatrics 2007;119:1113-19.

37. Leffler DA, Dennis M, Edwards George JB, et al. A simple validated gluten-free diet adherence survey for adults with celiac disease. Clin Gastroenterol Hepatol 2009;7:530-6, 536.e531-2.

38. Varni JW, Curtis BH, Abetz LN, et al. Content validity of the PedsQL 3.2 Diabetes Module in newly diagnosed patients with type 1 diabetes mellitus ages 8-45. Qual Life Res 2013;22: 2169-81.

39. Wherrett D, Huot C, Mitchell B, et al. Type 1 diabetes in children and adolescents. Can J Diabetes 2013;37(Suppl 1):S153-62.

40. International Diabetes Federation ISfPaAD. Other complications and associated conditions. Global IDF/ISPAD Guideline for Diabetes in Childhood and Adolescence. 2011:124-8.

41. American Diabetes Association. Standards of medical care in diabetes-2014. Diabetes Care 2014;37(Suppl 1):S14-80.

42. Husby S, Koletzko S, Korponay-Szabo IR, et al. European Society for Pediatric Gastroenterology, Hepatology, and Nutrition guidelines for the diagnosis of coeliac disease. J Pediatr Gastroenterol Nutr 2012;54:136-60.

43. NICE. Coeliac disease. Recognition and assessment of coeliac disease. NICE clinical guideline. 2009:86.

44. Ludvigsson JF, Bai JC, Biagi F, et al. Diagnosis and management of adult coeliac disease: guidelines from the British Society of Gastroenterology. Gut 2014;63:1210-28.

45. Sud S, Marcon M, Assor E, et al. Health professional attitudes regarding celiac disease screening and treatment in pediatric type 1 diabetes. Can J Diab 2011;35:334-9. 